\title{
Prácticas empresariales con potencial de vinculación territorial: el caso del sector forestal chileno ${ }^{1}$
}

\author{
Francisco Gatica Neira ${ }^{2}$
}

\begin{abstract}
RESUMEN
Este trabajo busca identificar en qué medida se conectan las empresas forestales con las redes presentes en el territorio, ya sea con las organizaciones empresariales, sociales y del sector público a partir de algunas prácticas empresariales. El estudio se realiza a partir de los resultados generados en la aplicación de una encuesta a 55 plantas forestales de la zona Centro Sur de Chile, cubriendo al $71 \%$ del total censal, se realiza un análisis previo de datos y una regresión logística binaria para identificar las prácticas empresariales con mayor potencial eslabonador. Los resultados buscan entregar antecedentes que orienten mejor las políticas públicas en el territorio.
\end{abstract}

Palabras clave: Industria forestal, prácticas empresariales, redes territoriales.

\begin{abstract}
This paper seeks to identify how much the forestry companies are connected with networks in the territory, as well as with business organizations, social and public sector from a regions and communities level and from a certain business practices. The study is based from the results generated in the implementation of a survey of 55 forest plants in the south central Chile, covering $71 \%$ of the total census, is performed preliminary analysis of data and a binary logistic regression to identify business practices with the greatest potential links. The results seek to provide background information that orientate better the public policies in the territory.
\end{abstract}

Key words: Forest industry, business practices and local networks.

1 Proyecto Kawax-CONICYT. Artículo recibido el 26 de enero de 2011, aceptado el 4 de enero de 2012 y corregido el 3 de febrero de 2012.
2 Departamento de Economía y Finanzas, Universidad del Biobío (Chile). E-mail: fgatica@ubiobio.cl 
El sector forestal está actualmente en una etapa de consolidación exportadora por la vía de la diversificación de mercados y por el desarrollo de la industria de remanufactura (tableros, enchapados, otros), con una alta concentración de la propiedad del suelo en dos compañías, generando externalidades que afectan la probabilidad de que otra actividad innovadora pueda emerger en el territorio (por ejemplo, la industria del mueble).

Un elemento clave para el desarrollo del país pasa por vincular virtuosamente las cadenas exportadoras con su entorno. En este contexto, el sector forestal chileno esta gradualmente pasando de ser un enclave productivo, caracterizado por una alta integración vertical y una elevada internalización de las actividades en la planta, a constituirse en pirámides productivas donde se generan un conjunto de relaciones de subcontratación en labores con complejidad creciente pero con alta una verticalidad en los poderes de negociación (Scott, 1998)

Las relaciones de las empresas forestales con su entorno productivo están fuertemente determinadas por el actual marco de incentivo, el que está centrado en alcanzar menores costos de producción y un limitado riesgo operacional por la vía del tamaño, el control de los insumos principales y la diversificación de mercados, más que por la vía de la innovación de productos y diseño al interior de la cadena (Katz et al., 1999).

Este trabajo comienza presentando un breve marco conceptual sobre los diferentes tipos de innovación, la idea de las pirámides productivas y el desarrollo endógeno. Posteriormente se entrega una visión de la cadena, se presentan las diferentes fases productivas y se desarrollan algunos rasgos estructurales relevantes desde la perspectiva territorial. En un tercer punto se desarrolla la metodología definiendo las variables y los instrumentos de análisis.

El estudio de campo comienza revisando el comportamiento de la variable tasa de innovación y nivel de conexión a redes según subsectores y grupos de comunas (territorios forestales). Luego se identifican, mediante una regresión logística binaria, las prácticas empresariales que aumentan o disminuyen la probabilidad de que una planta se conecte de forma más intensiva a las redes locales.

El énfasis de este trabajo no está en indagar el tipo de vinculación que se dan al interior de las redes productivas (insumo-producto), sino que se busca identificar en qué medida las empresas forestales pertenecen a una red territorial integrada por organizaciones sociales, empresariales y del sector público, con el objetivo de identificar aquellas prácticas que tienen mayor potencial eslabonador, acercando las empresas forestales con los actores del territorio.

\section{Tipos de innovación, estructura productiva piramidal y redes territoriales}

Este trabajo se levanta sobre tres ejes de análisis: los diferentes tipos de innovación y su capacidad difundir estímulos en el territorio, la presencia de estructuras productivas piramidales y la existencia de redes locales como un elemento clave para entender el desarrollo endógeno.

Un primer eje de análisis es identificar las fuerzas que están detrás del binomio innovación y territorio (Breschi \& Malerba, 1997). Existen dos tipos de regímenes de innovación que tienen un impacto heterogéneo en el territorio, y que surgen de la combinación de diferentes rasgos estructurales del mercado ${ }^{3}$ :

El primero es conocido como el "modelo amplio" (Shumpeter I) de bajos niveles de concentración industrial, baja estabilidad y alta natalidad de empresas, cuestión que se

\footnotetext{
3 Estos rasgos estructurales de mercado explicarían diferencias intersectoriales de la innovación, con distintas oportunidades tecnológicas, condiciones de apropiabilidad y acumulación del conocimiento, impactando en la difusión territorial del cambio técnico. Al respecto, Granda (2003) explica los elementos que constituyen un régimen tecnológico: i) las diferentes fuentes de conocimiento y los múltiples procesos de aprendizaje disponible, siendo relevante la condición de público del conocimiento, ii) las oportunidades tecnológicas que se refiere a la posibilidad o facilidad para alcanzar una innovación, iii) el grado de apropiabilidad de los resultados mediante el esfuerzo de innovación, y iv) el carácter acumulativo de la innovación.
} 
explica por un conocimiento con altas oportunidades tecnológicas, baja apropiabilidad y acumulatividad. En el territorio este régimen tendería a presentar una amplia difusión de la innovación por la vía de diferentes redes locales, lo que acorta las brechas tecnológicas y densifica el tejido productivo local.

El segundo, es el "modelo profundo" (Shumpeter II) caracterizado por una mayor concentración industrial, un aumento en el tamaño de los innovadores, estabilidad al interior del mercado y una baja natalidad, cuestión que se explica por elevadas condiciones de oportunidad, alta apropiabilidad y fuerte acumulatividad tecnológica. En este modelo la brecha tecnológica sería creciente, sin que existan procesos de difusión entre un polo innovador y el resto de la economía local.

En definitiva, de darse el "modelo profundo", el desarrollo tecnológico del sector forestal no se difundiría al resto de las empresas que están a su alrededor, reforzándose la condición de "enclave" productivo en territorios precarios.

Un segundo eje es el desarrollo de pirámides productivas (Scott, 1998, Storper y Harrison, 1994). Estamos hablando de una organización productiva caracterizada por un "núcleo empresarial" y un conjunto de subcontratistas que le prestan servicio en una relación de poder desigual. La estructura permite, a partir de confianzas intermedias, aprovechar las diferencias de costos de producción, la posibilidad de distribuir los riesgos operacionales-financieros, y la posibilidad de complementar competencias para contar con un producto con un mayor grado de diferenciación, calidad y menor precio.

En este tipo de estructura es clave la definición de algunos estándares de calidad y seguridad que permitan las relaciones de insumo-producto (normas del tipo ISO 9000), una base de optimización dentro de los eslabones de la cadena (modelos de justo a tiempo) y los procesos semicontinuos de producción que faciliten la fragmentación de una parte de la cadena para su externalización. A partir de esto, el sector forestal chileno presentará en el futuro una organización más compleja de la producción en el territorio, generando redes de subcontratistas, con un mayor grado de competencias y horizontalidad.

Un tercer eje de análisis es la relación entre prácticas empresariales y las redes territoriales. En este contexto, surge el modelo del desarrollo endógeno que plantea que los territorios que experimentan un mayor nivel de desarrollo son aquellos que presentan cuatro fuerzas que actúan en forma sinérgica: a) un desarrollo urbano a partir de la oferta de espacios públicos, zonas industriales y tecnológicas, b) una organización productiva flexible capaz de responder a los múltiples requerimientos al nivel de tamaño y especificidades de parte de los clientes, c) el desarrollo de instituciones entendidas como los espacios de construcción de confianzas, lo que reducen de los costos de transacción y permiten la generación de alianzas, y d) la difusión de la innovación, cuestión que requiere de un conjunto de redes en el territorio que permitan difundir e incrementar el conocimiento original (Vázquez-Barquero, 2004; 2006). En este contexto, las empresas competitivas se articulan alrededor de algunas prácticas empresariales, con diferentes redes territoriales, las que se mueven en cada fuerza del desarrollo endógeno.

La importancia de vincular cadena productiva y territorio no solo responde a la necesidad de tener un mayor nivel de igualdad, sino también es clave en la sustentabilidad de largo plazo del sector forestal chileno, asunto que obliga a pensar en forma paralela la "innovación empresarial" con la "innovación social", siendo esta última la que tiene efectos sobre la cohesión social, el reforzamiento de la imagen local, la vinculación con instituciones, la valoración de la formas de capital y finalmente la mejora del bienestar (Caravaca et al., 2005)

Este trabajo busca identificar en qué medida se conectan las empresas forestales con las redes presentes en el territorio, a partir de algunas prácticas empresariales. Lo anterior permite que las políticas públicas adapten las mezclas de iniciativas a cada realidad local (Fukuyama, 2004). Esto implica mirar "caso a caso" los factores que pueden generar una rearticulación, recogiendo así la variedad de situaciones, en el contexto de una "lectura 
del territorio", que sustente otras formas de governance local (Carmona, 2006).

\section{Visión de la cadena y del territorio}

Al analizar el binomio cadena y territorio se comprueba un alto nivel de heterogeneidad en los distintos tipos de desarrollo. La posibilidad de encontrar redes territoriales al nivel de organizaciones sociales, empresariales y comunales cambia en función de la localización de partes específicas de la cadena. Las condiciones serán distintas de acuerdo al contenido de conocimiento versus rutina que tiene en las actividades que están en el territorio (Pérez, 2010). Esta variedad define la necesidad de contar con políticas públicas para cada caso.
Diferentes eslabones de la cadena y su especialización territorial

Encontramos tres grandes actividades al interior de la cadena (Figura $\mathrm{N}^{\circ} 1$ ) con la consiguiente vinculación territorial.

Actividad silvícola: que va desde las labores de mejoramiento genético de la semilla, hasta la explotación del bosque. Esta actividad es la más extensa desde el punto de vista geográfico. En su expansión ha generado un conjunto de externalidades: procesos migratorios, disminución de la biodiversidad, mayor flujo de camiones aumentando la probabilidad de accidentes, entre otras externalidades.

La localización de esta actividad se da principalmente en comunas de la cordillera de la Costa y Nabuelbuta; con altos niveles

Figura $\mathrm{N}^{\circ} 1$

Principales eslabones de la cadena forestal

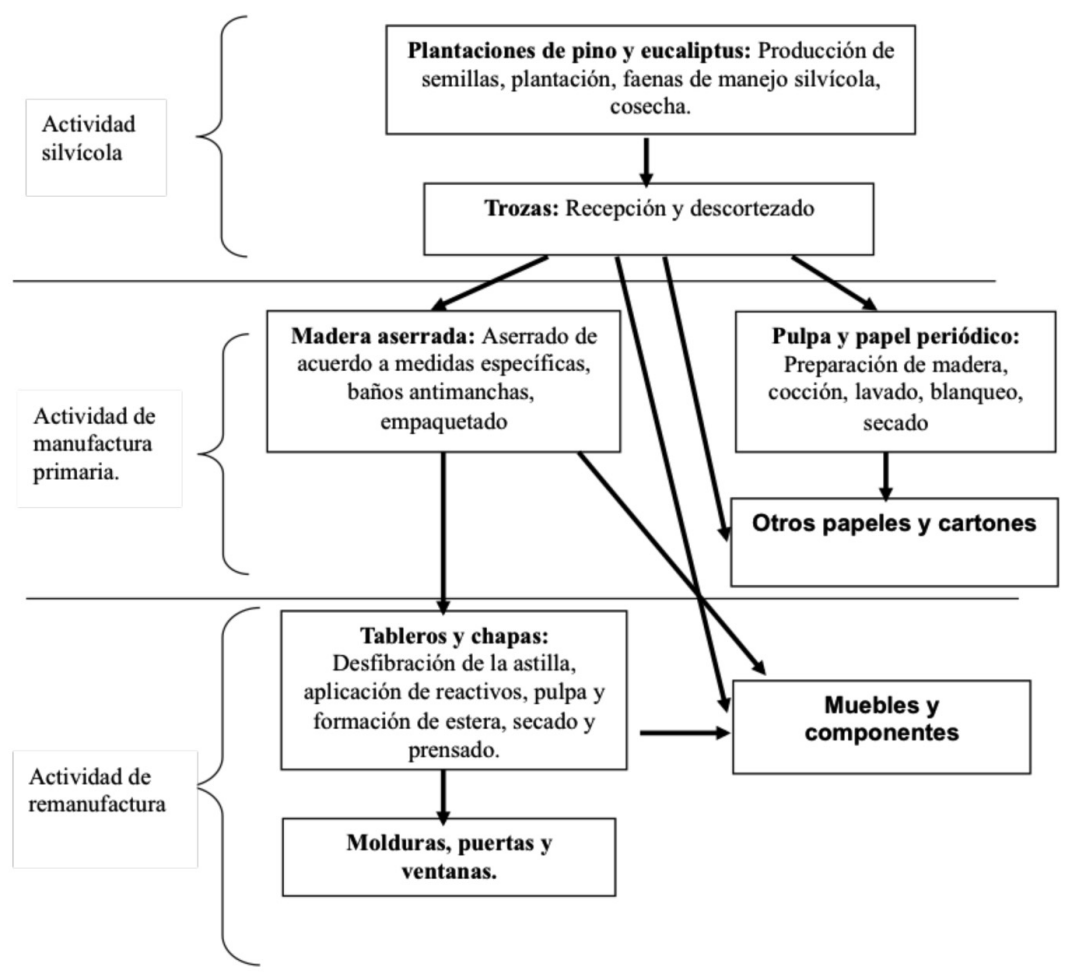

Fuente: basado en Katz et al., 1999, y Galdames, 2009. 
de pobreza, economías monoproductoras, con una notoria fuga de los jóvenes del territorio y un claro envejecimiento de la población. En definitiva la expansión forestal, en esta fase silvícola, ha generado un largo desencadenamiento de las antiguas redes agrícolas en el territorio (Guerrero, 2010).

Actividad de manufactura primaria: por un lado tenemos los aserraderos, los que actualmente experimentan un proceso de contracción, y la producción de celulosa que se consolida como la columna vertebral del sector. Encontramos fábricas de gran tamaño con una alta intensidad en capital. Su localización se concentra en Arauco, Nacimiento, Constitución, Valdivia, Itata, Laja, y en menor escala de producción algunas plantas pequeñas localizadas en las capitales regionales, por ejemplo en el Gran Concepción. En definitiva vemos dos trayectorias. Por un lado, un aserradero "antiguo", con un alto nivel de implantación territorial, integrado por empresas medianas, con un empresario local, con tecnología antigua y que tiene estrechos márgenes de comercialización, y por otro, los "grandes complejos forestales", que se asimilan a enclaves, que pertenecen a dos grandes grupos económicos, con tecnología de frontera, y principalmente dedicadas a la exportación. Ambas trayectorias coexisten generando un "Desarrollo Dual" (Fallabella, 2002).

Actividad de remanufactura: incluye tableros, aglomerados con diferentes densidades. Es una actividad "emergente" de la década de los noventa y surge como un proceso de integración vertical "hacia adelante". En la mayoría de las ocasiones estas empresas se ubican al interior del complejo forestal (al lado de la celulosa). La alta oferta de pino, plantada en la década de 1970, explica que los grandes conglomerados, como Arauco y CMPC, hayan experimentado un proceso de eslabonamiento productivo "hacia adelante" con productos como tableros y aglomerados (Silva y Moguillansky, 2001).

\section{Rasgos que determina el binomio cadena y territorio}

A partir de lo anterior se configuran algunos rasgos que condicionan el desarrollo de redes territoriales alrededor de la planta. a) Concentración en dos compañías y reducción de la variedad productiva: un elemento que condiciona las posibilidades de desarrollo es la alta concentración en la propiedad del suelo en dos grandes compañías, lo que dificulta los procesos de diversificación productiva (por ejemplo la industria del mueble). El actual marco de incentivo apunta al desarrollo concéntrico "hacia atrás" de la cadena de valor, permitiendo el mayor control de la propiedad del bosque. De esta forma se alcanzan economías de escalas con bajos riesgos operación. Todo lo anterior genera una tendencia a la monoproducción en el territorio. En esta línea para la Región del Biobío no observamos una diversificación de la canasta exportadora en el plazo de 20 años. El sector forestal en 1990 explicaba un $64 \%$ del total regional según valores US\$FOB y en el 2010 sube a un $74 \%$ (INE, 2010).

b) Lejanía de los espacios conversacionales: producto de la alta concentración en la propiedad de la empresa forestal, tenemos una mayor lejanía entre los actores territoriales y las gerencias generales de la empresa ubicada en la capital nacional. Lo anterior dificulta la posibilidad de generar una articulación público-privada en el territorio.

c) Asimetrías en las posiciones estratégicas: en función de la vecindad con cada parte de la cadena de valor. En general las intervenciones desconocen los diferentes tipos de inserciones que tienen los territorios en una misma cadena, siendo muy distinto contener a la actividad silvícola que una planta de remanufactura, $y$

d) Proceso de mayor concentración: la propiedad forestal en dos empresas obliga a trabajar la heterogeneidad al nivel de plantas debido a que se tiende a homogeneizar las tecnologías, el capital y las rutinas organizacionales, debiéndose recoger la específica historia de implantación territorial.

Este fenómeno de lejanía de los espacios conversacionales, explicado por la concentración entre la empresa y los actores locales, fue estudiado por Fløysand et al. (2010), para 
el caso de la salmonicultura y su relación con los municipios chilotes. Al respecto resulta interesante el concepto de "doble jerarquía" que enfrenta el municipio. Por un lado deben responder a los desafíos del país, definido al nivel del Estado-Nación, y por otro, deben resolver la demandas de sus comunidades locales, las que no siempre van en la misma dirección. En el caso de la industria del salmón, los autores concluyen, que el sistema local de innovación, entendido como el lugar donde se dan los procesos de aprendizaje sinérgico colectivo, parece ser el más débil.

\section{Metodología}

En principio se trabaja con la idea de planta-empresa como unidad de análisis. Se encuestan exitosamente a 55 empresasplantas del sector forestal, en el mes de enero de 2008 , cubriendo al $71 \%$ del total censal, a partir del directorio del Instituto Forestal (INFOR), este porcentaje de cobertura muestral permite extraer conclusiones con validez estadística. Un $62 \%$ de la muestra corresponden a aserraderos, que es consistente con la realidad productiva del sector. El $11 \%$ de las encuestas son plantas de celulosa y un $27 \%$ son empresas productoras de paneles (Cuadro $\mathrm{N}^{\circ} 1$ ).

La encuesta utiliza la escala likert de 1-5. Se evalúan diferentes aspectos en los ámbitos, como i) la estrategia empresarial y competitividad sistémica (42 preguntas), ii) densidad de las redes y orientación temática (13 preguntas), iii) tipos de innovación y avance técnico de las empresas (20 preguntas) y iv) factores que limitan la cooperación y la articulación (10 preguntas).

Cuadro $\mathrm{N}^{\circ} 1$

Empresas encuestadas

\begin{tabular}{|l|r|r|}
\hline & $\begin{array}{l}\text { Número } \\
\text { de } \\
\text { empresas }\end{array}$ & Porcentaje \\
\hline Total celulosa & 6 & 11 \\
\hline Total aserradero & 34 & 62 \\
\hline $\begin{array}{l}\text { Total paneles } \\
\text { (remanufactura) }\end{array}$ & 15 & 27 \\
\hline Total & 55 & 100 \\
\hline
\end{tabular}

Fuente: elaboración propia.
Principalmente se realizó un análisis de conglomerados estadísticos con el objetivo de aglutinar las variables que tienen un comportamiento similar, a partir de la consistencia conceptual. Se conglomeran variables a partir de los dendogramas (average linkage-between groups- con el paquete estadístico SPSS):

Para obtener la tasa de innovación se calcula un promedio de los parámetros de innovación: i) de productos, ii) de transporte y empaque, iii) de gestión, y iv) de diseño de producto. El análisis de conglomerados agrupa a estos parámetros en una distancia promedio $[5,10]$, porque presentan una distribución homogénea y consistente abarcando los diferentes ámbitos de la innovación en el sector forestal.

Para las conexiones a redes locales se determina un ratio a partir de promedios de las relaciones con las: i) organizaciones sociales, ii) organizaciones empresariales, y iii) el sector público. En términos generales el análisis conglomerado estadístico aglutina claramente los diferentes tipos de redes, con una distancia promedio $[5,10]$.

Por tanto, se define en un pool de diez variables, que responden a diferentes prácticas empresariales, que en términos generales presentan un bajo nivel de correlación entre sí. El par de datos que presentan mayor correlación es la dupla de inversión en certificación de competencias e inversión en actividad medioambiental $(\mathrm{rho}=0.6)$. Se probaron diferentes regresiones y la distorsión estadística de esta correlación es muy limitada, por tanto se opta por mantener ambas variables separadas

Hay que consignar que este trabajo no profundiza en el análisis longitudinal. Dicho de otra forma no evalúa parámetros como grados de centralidad, presencia de cliques, puntos de corte, variedad y vínculos débiles. Tampoco este análisis considera los elementos de "trasfondo" como son los sesgos e inercias culturales que condicionan los elementos de arquitectura de la red (Perrow, 1991; Hanneman, 2000)

Sobre este marco el análisis desarrolló dos niveles de trabajo: 
a) Una primera visión de las tasas de innovación y la conexión a redes locales a partir de las agrupaciones de comunas (territorios forestales) y subsectores industriales. Se busca identificar si hay un comportamiento distinto entre clasificaciones. Debido a que en algún grupo el número de empresas era muy pequeño se optó por no realizar una contrastación estadística, por tanto solo se grafican y se describen los principales resultados.

b) Una segunda visión se obtiene de los resultados del análisis de regresión logística binaria, con el objeto de identificar las prácticas empresariales que tienen mayor potencial explicativo en la probabilidad de conexión de las plantas con las redes territoriales.

En principio se intentó realizar una regresión lineal múltiple. En general la variable dependiente no sigue una distribución normal (prueba Kolmogorov-Smirnov; Stc=0.144, $\mathrm{Df}=55$; Sig=0.006). Producto de lo anterior, se optó por desarrollar un modelo de regresión logístico binario, llegando a relación 0/1 las diferentes intensidades en la vinculación de las redes. Cabe consignar que este tipo de modelo no enfrenta los supuestos estrictos de la regresión lineal múltiple (Hair, 1999).

En el Cuadro $\mathrm{N}^{\circ} 2$ se presenta en forma resumida las principales variables indicando el tipo de relación esperada. El lector comprobará que se utilizan dos niveles de exigencias en la definición binaria de la conectividad de las empresas forestales con las redes territoriales. Por tanto se generan dos modelos de regresión:

Una primera regresión más "exigente" donde las empresas que tengan nota promedio 4 y 5 en la intensidad de los contactos califican como conectadas con las redes sociales (valor=1) (Redtbo 5).

El otro modelo es "menos exigente" donde una empresa tiene redes territoriales cuando el promedio de la nota de vinculación con redes sociales es de un 3, 4, 5. (Redtbo 4).

Se probaron otras combinaciones para reclasificar la presencia de las redes sociales, pero los demás modelos resultaron con poca robustez estadística.

\section{Revisión previa de los datos}

A continuación realizaremos una descripción por agrupamiento sectorial y por comunas de las variables tasa de innovación y red total (no binaria).

\section{Visión sectorial}

Una primera revisión consiste en la comparación de los promedios de las diferentes variables analizadas según subsector industrial (celulosa, aserradero y paneles). Lo anterior busca identificar un bloque empresarial que presente un comportamiento significativamente distinto.

En principio, la variable nivel de conexión con la "red total" (parámetro dependiente) no presenta diferencias significativas al nivel de subsectores industriales ${ }^{4}$. En términos generales las notas fluctúan entre el 3.4 y el 3.8 . Esta constatación resulta interesante debido a que se descarta que exista una relación entre subsectores y una mayor propensión al contacto social, siendo las diferencias observadas marginales. Las empresas de celulosa y paneles presentan un mayor nivel de contacto social, pero con poca significancia estadística.

Algo distinto sucede con la variable "tasa de innovación global". Aunque no tenemos diferencias significativas vemos que las empresas del sector paneles presentan un mayor nivel de intensidad innovadora, en cambio las empresas de celulosa tienen un menor nivel de innovación, lo que es consistente con las edades tecnológicas de ambas líneas productivas.

En definitiva, las agrupaciones sectoriales no presentan un comportamiento significativamente distinto en los contactos territoriales e innovación total. Por tanto, el enfoque

4 Prueba $T$ student. Pensada para tamaños pequeños. $\mathrm{T}=((\mathrm{X}-\mu) /(\mathrm{s} / \sqrt{ } \mathrm{N}-1))$, donde la prueba $t$ devuelve la probabilidad asociada a que dos muestras pueden proceder de dos poblaciones subyacentes con igual media 
Cuadro $\mathrm{N}^{\circ} 2$

Presentación de variables utilizadas

\begin{tabular}{|c|c|c|}
\hline $\begin{array}{l}\text { Variable a explicar. } \\
\text { Probabilidad de tener } \\
\text { contacto con redes en } \\
\text { el territorio }\end{array}$ & $\begin{array}{l}\text { Variable independiente. } \\
\text { Prácticas o soluciones } \\
\text { empresariales con conte- } \\
\text { nido estratégico. }\end{array}$ & $\begin{array}{l}\text { Relación hipotética. Relación preliminarmente positiva } \\
\text { en la vinculación de la práctica o solución empresarial y } \\
\text { la probabilidad de contacto con redes territoriales. }\end{array}$ \\
\hline $\begin{array}{l}\text { Contacto con redes } \\
\text { en el territorio }(0 / 1) \text {. } \\
\text { Redes territoriales en } \\
\text { los ámbitos más meso } \\
\text { del desarrollo: orga- } \\
\text { nizaciones del sector } \\
\text { público, sociales y } \\
\text { empresariales }\end{array}$ & $\begin{array}{l}\text { Incorporación de mano } \\
\text { de obra de baja comple- } \\
\text { jidad (INCMOBA) } \\
\text { Incorporación de mano } \\
\text { de obra de alta compleji- } \\
\text { dad (INCMOCOM) } \\
\text { Nuevos contratistas } \\
\text { (NUCONTES) } \\
\text { Tasa de innovación glo- } \\
\text { bal (INNTO2) }\end{array}$ & 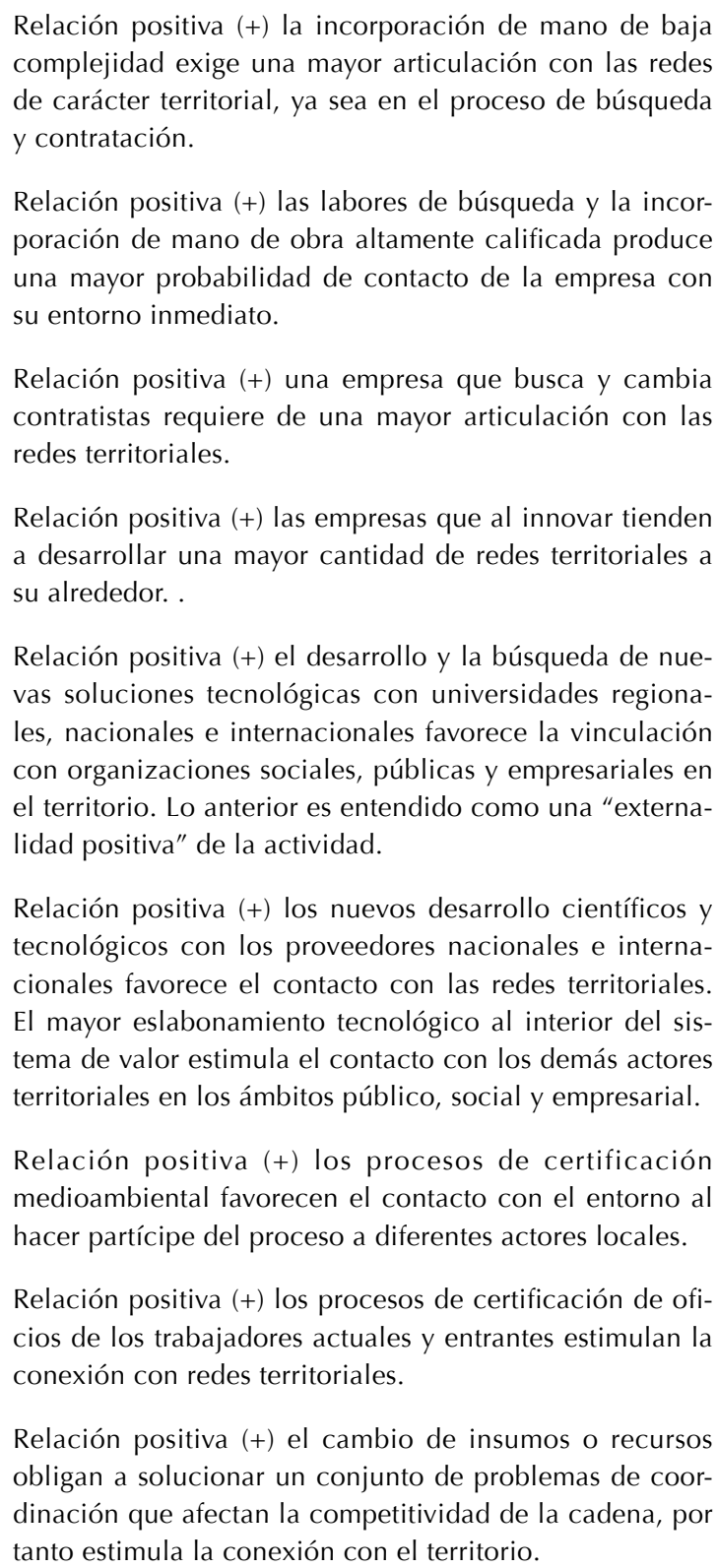 \\
\hline
\end{tabular}

Fuente. Elaboración propia. 
tradicional de la política industrial no logra recoger la variedad en la relación tasa de innovación y densidad de los contactos alrededor de la planta. De aquí que este trabajo apunte a relacionar cadena productiva y territorio específico, por ser un nivel donde la política pública puede actuar "caso a caso", para estimular el mayor contacto social y el aprovechamiento o mitigación de las externalidades en el espacio local.

\section{Visión territorial}

La agrupación de comunas se realizó guardando el criterio de vecindad geográfica. Debido a que hay agrupaciones que tienen muy pocas empresas no es factible realizar comparaciones de medias que sean de calidad estadística.

Para efectos de desarrollar este punto se presenta un mapa con los territorios forestales (Figura $\mathrm{N}^{\circ} 3$ ), se presenta el comportamiento de las tasas de innovación global y de vinculación social para cada agrupación (Figura $\mathrm{N}^{\circ}$ 4) y complementariamente, para cada caso, se entrega la población estimada (INE, 2010), los niveles de pobreza (CASEN, 2006) y el porcentaje de la población rural (INE, 2010).
Estos últimos datos se obtienen del Sistema de Información Municipal.

En el caso de Chillán, Chillán Viejo y Bulnes encontramos empresas pequeñas y medianas, ubicadas en ámbitos urbanos o periurbanos, que tienen una baja tasa de innovación y contacto social. Cabe consignar que este grupo de comunas tienen una población de 226.326 habitantes, con una tasa de ruralidad baja $(11,4 \%)$ y con una tasa de pobreza del $17,9 \%$, por debajo de la media del sistema estudiado (Cuadro $N^{\circ} 3$ ).

Presentan un alto promedio de innovación y contacto local un conjunto de empresas que están en Cabrero, Yungay y Tucapel. Esta agrupación tiene una población estimada de 61.208 habitantes, donde el $29.2 \%$ es rural, con una tasa de pobreza levemente superior del $19.9 \%$. Aquí se localizan unidades dedicadas a la producción de tableros y otras remanufacturas, plantas que pertenecen a los grupos CMPC y CELCO principalmente.

Por el lado de la vinculación con redes totales comprobamos que las actividades se mueven entre un 2.71 a un 4.11. Las empresas que tienen menor contacto con las redes

Figura $\mathrm{N}^{\circ} 2$

Grupo de empresas según subsector. Tasa de innovación global promedio y tasa de conexión a red global promedio

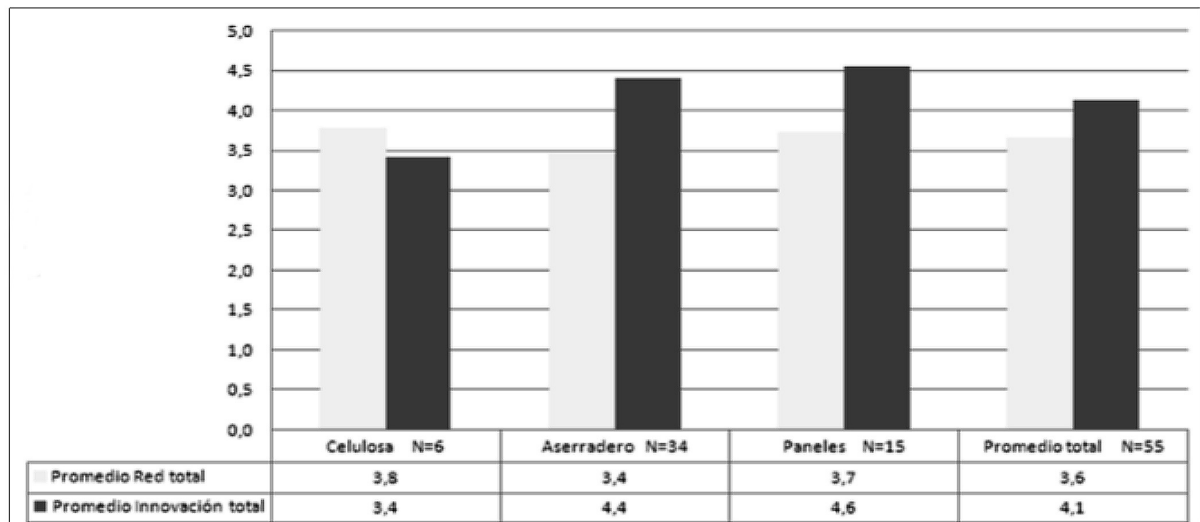

Fuente: Elaboración propia. 
Figura $N^{\circ} 3$

Territorios forestales a partir de agrupación de comunas

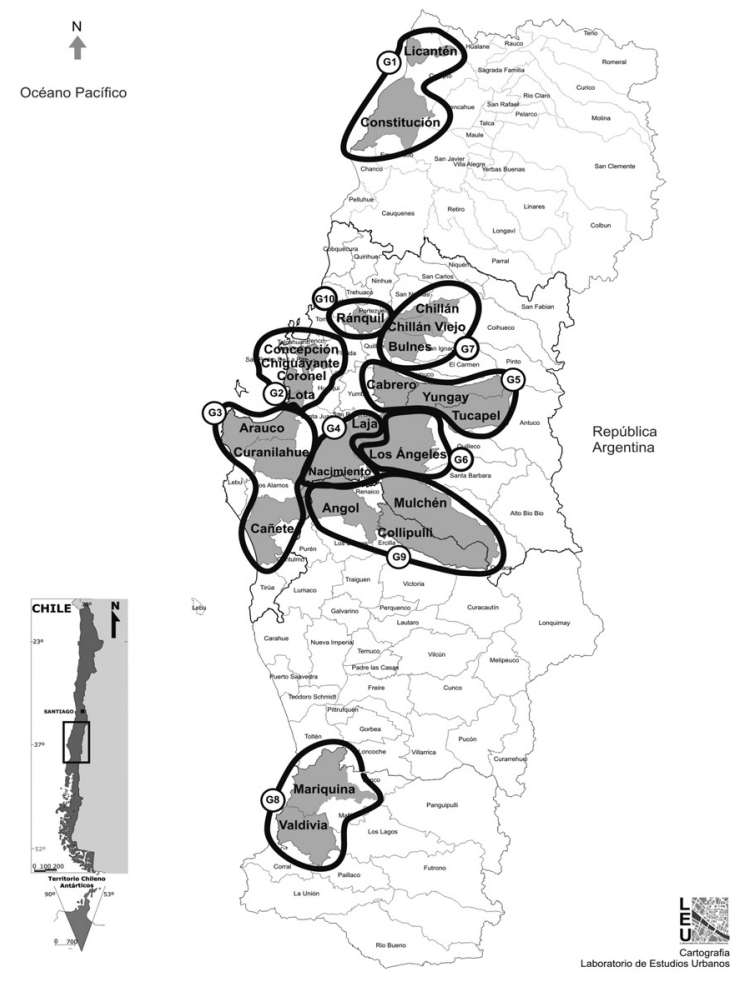

Fuente: Laboratorio de Estudios Urbanos, Universidad del Bío-Bío, 2012.

Figura $\mathrm{N}^{\circ} 4$

Grupos de empresas forestales en el territorio. Número de empresas, tasa de innovación global promedio y tasa de conexión a red global promedio

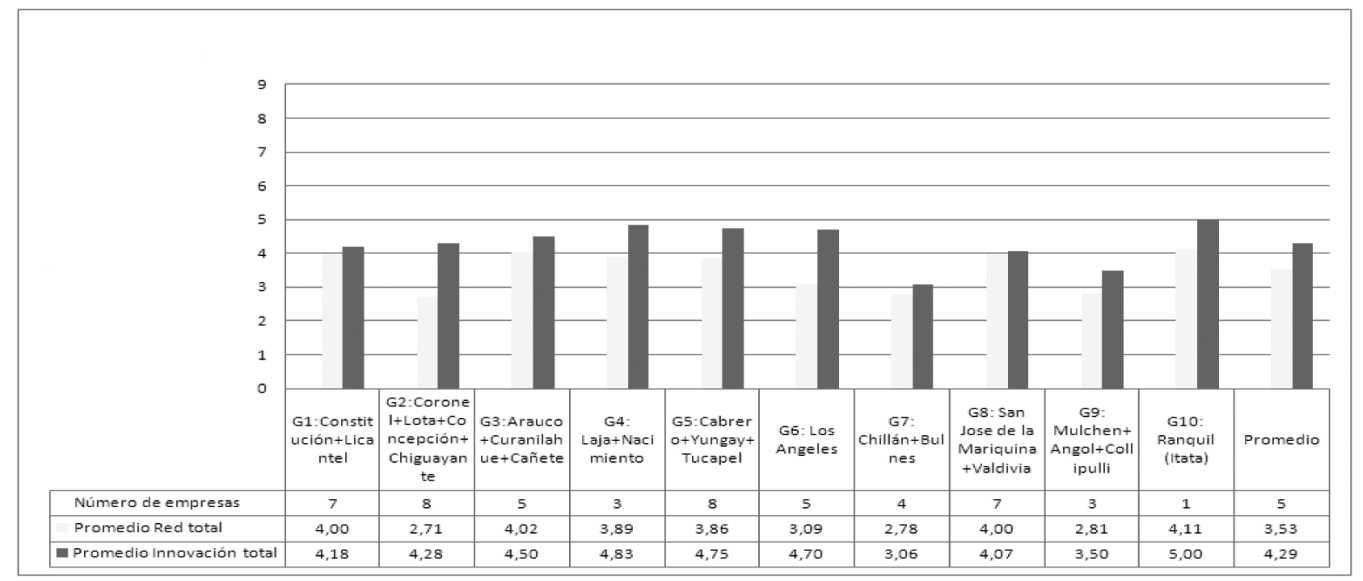


sociales locales son aquellas ubicadas en las comunas de Coronel, Lota, Concepción y Chiguayante. Esta zona es densamente poblada con 468 mil habitantes, de los cuales $97,7 \%$ vive en ciudades con niveles de pobreza del $17,6 \%$. En general contiene un grupo de empresas medianas y pequeñas, las que no tendrían mayores necesidades de articulación a una red territorial. Resulta interesante constatar que en el Gran Concepción se localizan una parte de las gerencias de los dos principales grupos empresariales que participan en el sector forestal (CELCO y CMPC).

En la misma línea tendríamos las plantas que están en la comuna de Los Ángeles, donde el contacto con las redes sociales es relativamente bajo (3.09), manteniendo un nivel interesante de innovación (4.70), este territorio presenta niveles de pobreza por debajo de la media ( $17,3 \%$ de su población), predominando la localización de los aserraderos.

En términos generales las agrupaciones que tienen un mayor nivel de vinculación con redes territoriales son las que contienen los principales complejos industriales:

- Ranquil, que contiene el Complejo Forestal Itata, que corresponde a una gran empresa de propiedad de CELCO, con tecnología de punta, ubicada en una zona rural, y que integra el aserrío y celulosa ${ }^{5}$. Esta es una comuna pequeña de 4.983 habitantes, ubicada en la cordillera de la Costa, donde el $80,8 \%$ de la población es

5 Resulta interesante el análisis realizado por Mendez y Ondategui (1999) sobre el caso de Madrid (España). En este trabajo los autores constatan que producto de la reconversión industrial hay una creciente inestabilidad espacial: por un lado tenemos las empresas de localizadas en las zonas urbanas se mueven en segmentos maduros y limitadas debido a que no tienen posibilidades de crecimiento. Por otro, tenemos la localización a las "afueras" de la ciudad, en nuestro caso en la zona rural, próximos a las grandes vías de transportes (carreteras), las empresas se caracterizan por alcanzar mayores economías de escala, tienen actividades más modernas y presentan por tener un mayor potencial innovativo.

Cuadro $\mathrm{N}^{\circ} 3$

Población, pobreza y ruralidad en territorios forestales

\begin{tabular}{|l|r|r|r|}
\hline Territorios forestales & \multicolumn{1}{l|}{\begin{tabular}{l} 
Población \\
\multicolumn{1}{l|l|}{$\begin{array}{l}\text { Porcentaje } \\
\text { de población } \\
\text { rural }\end{array}$}
\end{tabular}} & $\begin{array}{l}\text { Porcentaje } \\
\text { de población } \\
\text { pobre }\end{array}$ \\
\hline G1: Constitución + Licantén & 60.638 & 26,8 & 21,9 \\
\hline G2: Coronel + Lota + Concepción + Chiguayante & 498.687 & 2,3 & 17,6 \\
\hline G3: Arauco + Curanilahue + Cañete & 105.543 & 22,8 & 23,3 \\
\hline G4: Laja + Nacimiento & 47.109 & 25,2 & 23,1 \\
\hline G5: Cabrero + Yungay + Tucapel & 195.813 & 29,2 & 19,9 \\
\hline G6: Los Ángeles & 226.326 & 25,5 & 17,3 \\
\hline G7: Chillán, Chillán Viejo + Bulnes & 177.129 & 11,4 & 17,9 \\
\hline G8: San José de la Mariquina + Valdivia & 101.358 & 14,0 & 15,7 \\
\hline G9: Mulchén + Angol + Collipulli & 4.983 & 80,8 & 25,9 \\
\hline G10: Ranquil (Itata) & 1.478 .794 & 13,8 & 8,7 \\
\hline Totales & & & 18,8 \\
\hline
\end{tabular}

Fuente: Elaboración propia a partir del Sistema de Información Municipal, 2012; INE, 2010; CASEN, 2006. 
rural y sus tasa de pobreza es del $8.7 \%$, siendo la más baja del territorio estudiado.

- Arauco, Curanilahue y Cañete donde se localiza el complejo Arauco, Horcones I y II con sus fases de aserríos y producción de celulosa. El ámbito de influencia abarca una población de 105 mil habitantes, donde el $22,8 \%$ es rural y la pobreza $(23,3 \%)$ es relativamente alta.

- San José de la Mariquina, destacándose la planta de producción de celulosa dependiente de CELCO, con un ámbito de influencia que incluye Valdivia. Para la agrupación tenemos porcentaje de ruralidad y niveles de pobreza por debajo de la media del sistema.

- Licantén y Constitución donde hay un conjunto de empresas forestales de antigua data. En esta agrupación un 26,8\% de su población vive en zonas rurales y un $21,9 \%$ de sus habitantes que son calificados como pobres, valor que se mueve por sobre la media del sistema estudiado. En esta agrupación tenemos empresas de aserrío y celulosa.

- Laja y Nacimiento. En este caso la innovación es relativamente alta (4.83) y el nivel de vinculación a redes locales se mueve por sobre la media (3.89). Esta agrupación tiene niveles de pobreza por sobre la media del territorios forestales. Tenemos grandes plantas, vinculadas a los grupos empresariales, de gran influencia en lo urbano (company town).

- Por último, tenemos la agrupación de Mulchén, Angol y Collipulli donde tanto el promedio de innovación (3.5) y el de vinculación social (2.81) están por debajo de la media de los territorios analizados. Esta agrupación presenta niveles de pobreza y de población rural por sobre los valores promedios.

En definitiva, tanto para la agrupación de comunas como para la subsectores industriales las variables tasa de innovación total y vinculación con redes territoriales no presenta un comportamiento notoriamente diferente.
Frente a esto se realiza una regresión logística binaria para identificar en el total de 55 empresas aquellas prácticas, problemas o temas empresariales que explican un mayor nivel de vinculación territorial. En este sentido, solo considerando el ámbito de influencia alrededor de las plantas, los territorios forestales, contienen a 1.478 .794 de habitantes, los que en promedio tienen un $18.8 \%$ de pobreza. Por tanto, ante la alta variedad de situaciones surge la necesidad de tener una "mirada transversal" de los factores que pueden estimular la mayor vinculación empresa con las redes locales.

\section{Identificación de prácticas empresariales con potencial de vinculación territorial}

A continuación se presentan los resultados de un análisis de regresión logística binaria, con el objeto de identificar aquellas prácticas empresariales que tienen mayor potencial explicativo en la probabilidad de que una empresa-planta presente una conexión con las redes territoriales (Cuadro $\mathrm{N}^{\circ} 4$ ).

Tanto el modelo exigente (Redbto 5) como el menos exigente (Redbto 4) presentan ratios Nagelkerke ${ }^{6}-\mathrm{R}^{\wedge} 2^{\prime}$ de 0.33 y 0.58 . Al realizar la prueba Chi-cuadrado comprobamos que ambas regresiones tienen $\mathrm{R}^{\wedge} 2$ distintos de cero, con una significancia de 0.42 y 0.77 . Una primera constatación es que la cantidad de variables analizadas permite una explicación limitada del nivel de conexión territorial alrededor de las plantas. Por tanto, en un segundo trabajo, se debe aumentar la cantidad de parámetros a analizar, aumentando el universo de estudio, debido a que el tamaño de 55 empresas limita la potencia estadística afectando el $\mathrm{R}^{\wedge} 2$.

En términos generales, el modelo "menos exigente" (Redbto 4) en la definición del contacto (valor $1=3,4,5$ ), presenta una mayor capacidad de predicción, siendo su porcentaje de acierto (percent correct overall) cercano al $87 \%$. En cambio en el modelo exigente (Redbto 5), donde la definición de contacto

\footnotetext{
6 El ratio Nagelkerke $-R^{\wedge} 2$, similar en su interpretación al $R^{2}$ del análisis de regresión.
} 
tenía un valor 4 y 5 , el porcentaje de acierto es del $70.9 \%$

Al analizar los resultados de ambos modelos podemos distinguir aquellas prácticas empresariales que producen vinculación territorial de aquellos que, según los resultados, no tienen actualmente mayor incidencia en la construcción de redes territoriales.

\section{Las prácticas que no producen vinculación}

Un primer resultado es que la tasa de innovación (INNTO2) por sí sola no garantiza que las empresas vayan a tener un mayor contacto con el territorio. El tipo de innovación del sector forestal es del tipo "profundo" $y$, por tanto, "polar", pudiendo pronunciar aún más la presencia de los enclaves. Resulta interesante que este parámetro tenga una relación inversa $(B=-1.08)$, pero poco significativa, en su relación con la variable dependiente ( wald=2,05 y sig=0.15). En el fondo no hay una relación positiva, pero tampoco negativa, entre innovación y vinculación a redes territoriales.

Inclusive en la regresión "más exigente" (Redtbo 5) la variable innovación, pese a que es poco significativa ( $\operatorname{sig}=0.1)$, tiene un $B=-$ 0.93; cuestión que evidencia, para el grupo estudiado, existe una relación inversa entre innovación y conexión con redes territoriales, pero poco significativa.

Por tanto, a partir de las dos regresiones llegamos a la misma conclusión: a medida que la empresa forestal tiende a aumentar su tasa de innovación, no necesariamente tendremos un mayor nivel de vinculación con redes territoriales. De aquí que pensemos que las políticas sectoriales, por sí solas, no aseguren mejorar la actual distribución del ingreso en el territorio, configurando un desarrollo excluyente más que inclusivo.

Ambos modelos también permiten comprobar que el cambio en los contratistas (NUCONTES) no asegura la mayor conectividad con el territorio (en el caso del modelo Redtbo 4: $B=-0,4$; wald=1,25; sig=0.26). Se plantea la "hipótesis del horizonte limitado", (Oakey et al., 2001), donde las empresas al alcanzar un mayor tamaño y complejidad in- corporan en su proceso de gestión la búsqueda de contratistas fuera de la región.

En el caso forestal los procesos de innovación, centrado en la compra de tecnología en el extranjero, tienden a desplazar a los contratistas locales, producto de las brechas tecnológicas y la venta del paquete tecnológico completo en la figura de grandes contratos de ingeniería.

En la misma línea la actividad científica y tecnológica desarrollada con los proveedores (ATProvee) no asegura mayor vinculación territorial alrededor de la empresa $(B=-0.28$; wald $=0.53$, sig $=0.46$ ). En este sector la innovación está en parte determinada por los proveedores especializados internacionales. Por tanto los contratistas para labores de mantención de la tecnología provienen directamente del extranjero ${ }^{7}$.

En el caso del sector forestal chileno tendríamos presentes dos grandes taxonomías del cambio técnico (Pavitt, 1984). Por un lado tendríamos sectores intensivos en escalas de operación, los que pueden mantener laboratorios de I+D propios, en especial en el caso de los dos principales grupos empresariales (CELCO y CMPC). Por otro, el sector también estaría dominado por proveedores especializados, comprando tecnología ya desarrollada, en la forma de paquetes integrados que incluyen los servicios de mantención.

Finalmente, se constata que la incorporación reciente de mano de obra calificada (INCMOCOM) no produce una mayor vinculación con el territorio alrededor de la planta $(B=-0.3 ;$ Wald $=0.42 ;$ Sig=0.51). Estamos frente a procesos intensivos en capital. El flujo de mano de obra calificada proviene principalmente de la capital regional y nacional, lejana de la localización de la planta.

\footnotetext{
7 Este proceso de sustitución de los contratistas locales lo refleja muy bien el estudio de Katz (2000), quien identifica el proceso de sustitución de los proveedores locales con oferentes extranjeros de tecnologías, explicado por los bajos tipos de cambio y altos costos de mantención de la tecnología antigua. Esto afectó a los contratistas locales, dejándolos afuera del sistema de valor forestal.
} 


\section{Los factores que producen vinculación} con el territorio

En principio los resultados del "modelo poco exigente" revelan (Redbto 4) que a medida que es más reciente la incorporación de mano de obra poco calificada (INCMOBA) aumenta la probabilidad de tener vinculación con los actores en el territorio $(\mathrm{B}=0.78$; wald $=4.36$ y Sig 0.03). Lo anterior dice relación al tipo de industria que se está analizando. El sector forestal, además de ser intensivo en capital, requiere una cantidad importante de mano de obra poco calificada, obligando a articularse con un conjunto de organizacio- nes que giran alrededor de algunas oficinas públicas en el territorio (por ejemplo, municipalidades).

Otra constatación del modelo (Redbto 4) es que las empresas que hasta hace poco tiempo cambiaron recursos productivos o insumos (CAMINSU) presentan mayor probabilidad de contactos territoriales $(B=0.86$; wald 4.06 y sig 0.04). El cambio de insumos implica reordenar la cadena de producción y por tanto genera una mayor tendencia a coordinar mejor la red logística de cada empresa. A diferencia del cambio de contratista, el

Cuadro $\mathrm{N}^{\circ} 4$

Resultados de la regresión logística binaria

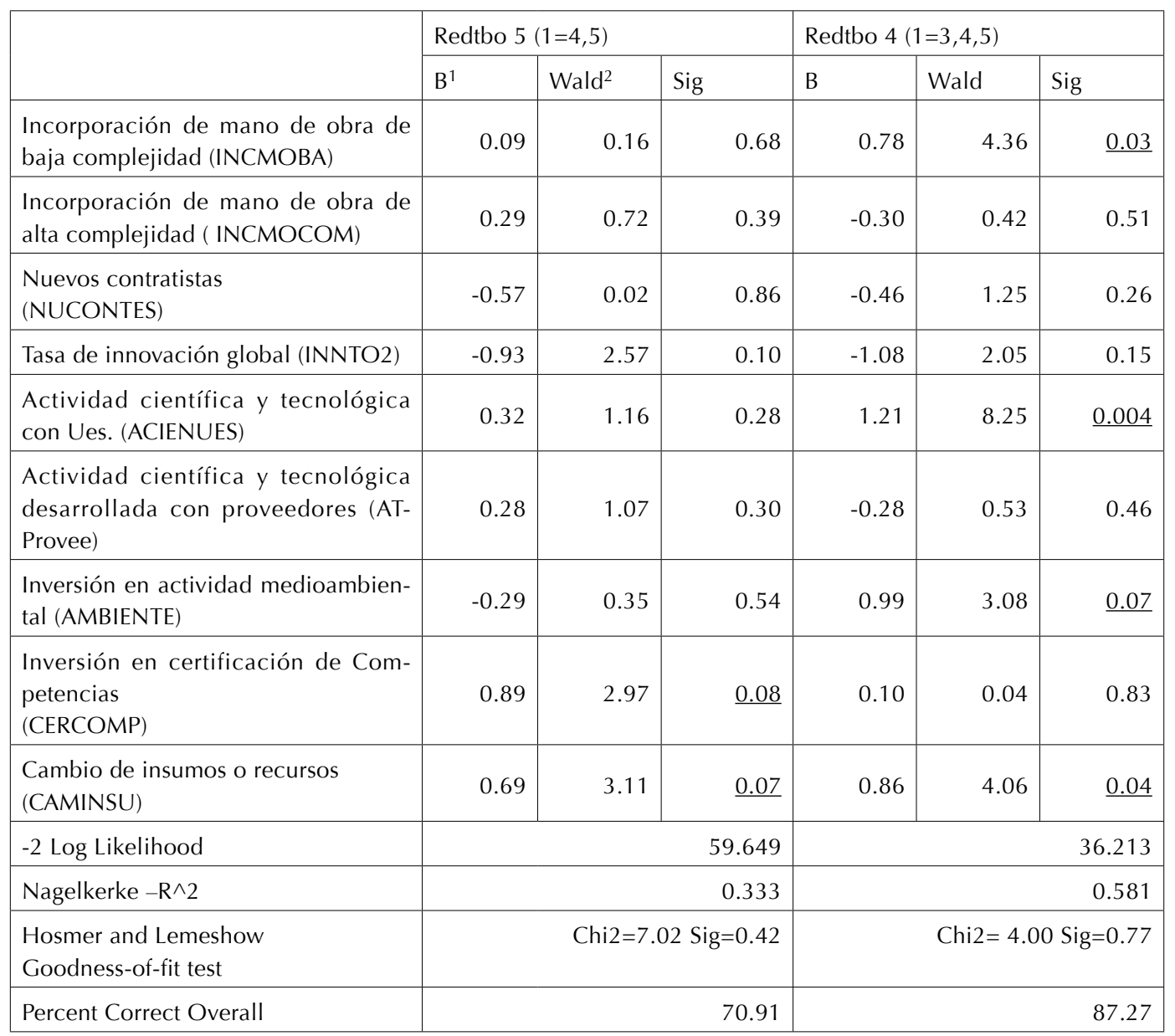

Fuente: Elaboración propia a partir de los resultados de la regresión. 
cambio en el tipo de insumo constituye una oportunidad para construir redes territoriales.

Por otro lado, la inversión en certificación medioambiental constituye un tema vinculante con el territorio (AMBIENTE). Producto de las presiones globales, los procesos asociados a la certificación ambiental obligan a contar constantemente de una articulación con redes territoriales. Esta vinculación es parte de la gestión empresarial y aparece como una fuerza que conecta con el territorio $(B=0,99$; Wald=3.08; Sig=0.07).

La variable certificación de competencias (CERCOMP) comienza a ser relevante una vez que aumentamos la exigencia sobre la definición de contactos (Redbto 5). Cuando corremos este modelo esta variable presenta $\mathrm{B}=0.89$, Wald=2.97, Sig= 0.08. La explicación de este resultado pasa por el hecho que la certificación de competencias responde a una necesidad de formalización de la mano de obra local, agregando un elemento diferenciador al mercado del trabajo. Por tanto, surge como un parámetro explicativo cuando evaluamos nivel de implantación territorial alrededor de la planta, con mayores niveles de exigencia.

Finalmente, el modelo poco exigente (Redbto 4) detecta como una oportunidad de conexión territorial, la posibilidad de mantener vinculación científica y tecnológicas con las universidades nacionales e internacionales (ACIENUES) $(B=1,21$; Wald=8.25 y $\mathrm{Sig}=0.004)$. Esto es interesante considerando que las tasas de innovación total no resultaba ser una variable significativa para el nivel de conexión territorial. Es probable que las universidades cumplan un rol de capacitador de la mano de obra y de asesoramiento en el proceso de adecuación y evolución de ciertos paquetes tecnológicos extranjeros a las especificidades y diagnósticos locales.

\section{Conclusión}

En definitiva tenemos un conjunto de parámetros entre los que se cuentan el tipo de innovación, el cambio en los contratistas, la actividad científica con proveedores y la incorporación de mano de obra calificada que no tienen una relación significativa con la probabilidad de conexión de las empresas forestales con las redes territoriales integrada por organizaciones sociales, empresariales y públicas.

Cabe consignar que estamos hablando de territorios precarios, no innovativos, que se alejan del paradigma de territorios como el Silicom Valley o la Ruta 128. Hablamos de zonas agrícolas y forestales que contienen labores basadas en la explotación de recursos naturales, con mano de obra poco calificada y altos índices de pobreza.

Frente a estos resultados surge la pregunta:

¿Apostar al desarrollo de clúster por la vía de la innovación concéntrica al interior de la cadena de valor forestal permite estimular el desarrollo del territorio por la vía a la conexión a redes locales?

Al parecer la respuesta no es tan clara. El tipo de innovación, ya sea amplio o profundo, determina la capacidad de conectar los conglomerados productivos con el entorno, reduciendo los actuales niveles de desigualdad. Por tanto, la sola profundización del actual modelo exportador puede llevar a un mayor crecimiento, un mayor ingreso per cápita, pero con una fuerte desigualdad y una baja movilidad social.

Sin embargo, los temas que abren oportunidades para la conexión con actores territoriales no responden a un "estado ideal", sino que son caminos más cercanos, pero de impacto en el entorno. Surgen variables como incorporación de mano de obra poco calificada, cambio de insumos, inversión en certificación de competencias, inversión en certificación medio ambiental y la actividad tecnológica con universidades.

En el fondo, los temas "vinculantes" con el territorio responden a "espacios cotidianos" que obligan a que las políticas públicas desarrollen a nivel territorial agendas específicas para cada situación, recogiendo y capitalizando aprendizajes clave al momento de pensar en la gestión. En definitiva hay que aceptar un nivel de "discrecionalidad" para desarrollar una mayor articulación socioproductiva. Los factores que son funcionales desde una lógica sectorial no necesariamente 
permiten aumentar la conexión territorial y por tanto alcanzar un mayor nivel de desarrollo.

Por tanto, se exige un "marco institucional flexible", donde cada territorio desarrolle una "agenda de conexión", bajo la modalidad de un "pacto territorial" (Carmona, 2006), donde con una lógica público-privada se actué de manera focalizada, desde abajo, en iniciativas de eslabonamiento entre las grandes empresas exportadoras y el tejido territorial. Lo que implica conocer muy bien las trayectorias de innovación y su potencial difusor en los tejidos locales, determinar de posibles puntos de contacto a partir de los temas que son "cotidianos" y que tienen impacto en la calidad de vida de la población.

Desarrollar un nuevo marco institucional es un paso previo para estimular las demás fuerzas del desarrollo endógeno, activando procesos de innovación más amplios que se difundan en el tejido local, alcanzando una organización productiva más compleja con pirámides basadas en la innovación y el aprendizaje, y finalmente generando un entorno más atractivo para vivir.

\section{Referencias bibliográficas}

BRESCHI, S. \& MALERBA, F. Sectorial Innovation Systems: Technological Regimes, Shumpeterian Dynamics, and Spatial Boundaries. In: Systems of Innovation Tecnologies, Institution and organization. London: Ed. Edquist Ch. Printer, 1997.

CARAVACA, I.; GONZÁLEZ, G. y SILVA, R. Innovación, redes, recursos patrimoniales y desarrollo territorial. EURE, 2005, Vol. XXXI, No 94, p. 5-24.

CARMONA, R. Los pactos territoriales e Italia. Un análisis de la experiencia reciente y sus implicancias en términos de governance. EURE, 2006, Vol XXXII, No 96, p. 117-133.

ENCUESTA DE CARACTERIZACIÓN SOCIOECONÓMICA NACIONAL (CASEN). La pobreza en Chile. Santiago de Chile: MIDEPLAN, 2006
FALLABELLA, G. ¿Se agotó el modelo?". FALABELLA, G. y GALDAMES, R. (editores). Repensar el desarrollo chileno. País, territorios, cadenas productivas. Concepción: Ediciones Universidad del Bío-Bío, 2002.

FLØYSAND, A.; BARTON, J. y ROMÁN, A. La doble jerarquía del desarrollo económico y gobierno local en Chile: el caso de la salmonicultura y los municipios chilotes. EURE, 2010, Vol. XXXVI, No 108, p.123-148.

FUKUYAMA, F. La construcción del Estado. Hacia un nuevo orden mundial en el siglo XXI. Barcelona: Ediciones B, 2004.

GALDAMES, R. (editor). Gran Empresa y territorio: El Sector Forestal Maderero Chileno del Maule a Los Ríos. Concepción: Ediciones Universidad del Bío-Bío, 2009.

GRANDA, I. Los regímenes tecnológicos schumpeterianos y su relación con la especialización tecnológica internacional: evidencia empírica. Madrid: tesis doctoral presentada en la Universidad Complutense de Madrid, 2003 (Inédito).

GUERRERO, R. El ocaso de la Sociedad Campesina. Concepción: Mimeografiado, Universidad del Bío-Bío, 2010.

HAIR, A. \& BLACK, T. Análisis Multivariado. Madrid: Prentice Hall, 1999.

HANNEMAN, R. Introduction to Social Network Methods. Los Angeles: Departament of Sociology, University of California, 2000.

INSTITUTO NACIONAL DE ESTADÍSTICAS (INE). Boletín de exportaciones regionales. Concepción: Región del Bío-Bío, 2010. Disponible en Internet: http://www.inebiobio.cl

INSTITUTO FORESTAL (INFOR). Distribución de las principales plantaciones forestales en el País, 2010. Disponible en Internet: http://www.infor.cl

KATZ, J.; STUMPO, G. y VARELA, F. El Complejo Forestal Chileno. Santiago de Chile: Documento de trabajo, CEPAL, 1999. 
KATZ, J. Pasado y presente del comportamiento tecnológico de América Latina. Santiago de Chile: serie de Desarrollo Productivo $N^{\circ} 75$, Red Reestructuración y Competitividad. CEPAL, 2000.

MÉNDEZ, R. y ONDATEGUI, J. La estructura territorial del sistema productivo. En: GARCÍA DELGADO, J. L. (editor). Lecciones de economía española. Madrid: Civitas, 1999.

OAKEY, R.; KIPLING, M. \& WILDGUST, S. Clustering among firms in the Non Broadcast Visual Comunication Sector. Regional Studies, 2001, Vol. 35, N 5, p. 401-414.

PAVITT, K. Sectoral Patterns of technical change: Towards a taxonomy and a theory. Research Policy, 1984, N 13, p. 343-373

PÉREZ, C. Dinamismo tecnológico e inclusión social en América Latina: una estrategia de desarrollo productiva basada en recursos naturales. Revista Cepal, N 100, 2010.

PERROW, CH. Sociología de las organizaciones. Madrid: McGraw-Hill, 1991.

SALA, I. y MARTIN, X. Apuntes de Crecimiento Económico. Antoni Bosch Editor, 2000.

SALA, I.; MARTIN, X.; BLANKE, J.; DRZENIEK, M. \& MIA I. The Global Competitiveness Index 2009-2010: Contributing to Long-Term Prosperity amid the Global Economic Crisis. The Global Competitiveness Report 2009-2010. 2009. New York: World Economic Forum.

SCOTT, A. J. Regions and the World Economy: The Coming Shape of Global Production, Competition, and Political Orden. Oxford: Oxford University, 1998.

SILVA, V. y MOGUILLANSKY, G. La voz de la Cepal: ¿Cómo se viene la mano? Revista Chile-Forestal, 2001.

STORPER, M. y HARRISON, B. Flexibilidad; jerarquía y desarrollo regional: Los cambios de estructura de los sistemas productivos industrial y sus nuevas formas de articulación del poder en los años 90. En: BENKO, G. y LIPIETZ, A. Las Regiones que ganan: Distritos y Redes. Los nuevos paradigmas de la geografía económica. Valencia: Edicions Alfons El Magnanin. Generalitat Valenciana, 1994.

VÁZQUEZ BARQUERO, A. Las Nuevas Fuerzas del Desarrollo. Barcelona: Antoni Bosch Editor, 2005.

VÁZQUEZ BARQUERO, A. Surgimiento y transformación de clusters y milieus en los procesos de desarrollo. EURE, 2006, № 95, p. 75-95. 
